\title{
SARS-CoV-2, COVID 19 Useful information
}

\author{
Juan Sebastián Espinosa-Serna* \\ Doctor of Internal Medicine, Hospital Universitario Mayor Méderi, Universidad del Rosario, Bogotá, Colombia
}

*Corresponding author: Juan Sebastián Espinosa-Serna, Doctor of Internal Medicine, Department of Critical and Itensive Care Medicine, Hospital Universitario Mayor Méderi, Universidad del Rosario, Bogotá, Colombia, Email: juansebastian_612@hotmail.com

\begin{tabular}{|c|c|}
\hline ARTICLE INFO & ABSTRACT \\
\hline Received: 幽 April 17, 2020 & Citation: Juan Sebastian Espinosa-Serna. SARS-CoV-2, COVID 19 Useful information. \\
\hline Published: 幽 April 28, 2020 & ad I Sci \& Tech Res 27(2)-2020 BISTR MS ID 004484 \\
\hline
\end{tabular}

\section{Questions That Officials and Institutions Should Be}

\section{Clear About}

a) Biosafety measures: protection for health personnel, location of patients with COVID-19

b) Define advanced management strategies

c) Define the medication to be used in the rapid intubation school

d) Mechanical ventilation management

a. What ventilation mode to use?

b. Use of protective mechanical ventilation parameters

c. When and how to put in a prone position?

d. What neuromuscular relaxant to use?

e) Patient route management for safe transfer to exams.

f) Bioethics and decision-making of ill patients with prognosis
1) How long do you wait to evaluate response?
g) Route of the corpses and handling of them.

\section{What is the Purpose of this Text?}

Provide the scientific community with a concrete and summary review of the subject of COVID-19 in relation to published articles; it does not symbolize a mandatory measure, it is a guide, an aid for this time of health crisis.

\section{Introduction}

SARS-COV-2, COVID-19 or Wuhan virus is the last RNA of the seven RNA viruses described whit affection in humans of the family
Coronaviridae subfamily Orthocoronavirinae of the order Nidovirals, characterized from the other positively charged RNA viruses (can be translated directly without the need for reverse transcriptase Baltimore III-mRNA + classification) as having the largest genome, so its cycle and transmission is not entirely clear.

In the Coronaviridae family there have already been two epidemics the first in 2002-2003 with SARS-CoV (Severe Acute Respiratory Syndrome) and the second with the MERS-CoV (Middle East Respiratory Syndrome), whose outbreak was in the 2012, with a mortality of approximately $11 \%$ and 35\% respectively, highlighting the high mortality that this group of viruses can have in humans [1,2]. Its name is derived from its microscopic shape similar to that of a solar corona and from the Latin corona, it has a wide distribution in the animal kingdom, mainly in mammals (porcines, felines, canines, humans, horses) and avian, considered a zoonotic infection, its clinical manifestations are mainly respiratory (from common flue to Acute respiratory distress syndrome -ARDS) and enteric symptoms (diarrhea), but in some cases neurological and liver involvement has been reported [1,2].

\section{Morphology, Structural Proteins and Access Path- ways}

The virus has a spherical shape, with a pleomorphic surface, its envelope is composed of several proteins, the S Protein or Spike proteins (mediate the binding of the virus with the cellular receptor, the $\mathrm{M}$ protein or membrane protein shapes the surface of the virus and the $\mathrm{E}$ or envelope protein that is part of the virus surface and has a role in the fusion of the virus with the cell to be infected, on average the virus has a diameter of 80-120 nm. Inside the envelope is the nucelocápside protein or $\mathrm{N}$ protein, which is responsible for maintaining the binding of the virus genome and 
protecting it. There are also accessory proteins or that complement the replication and function of the virus such as the HE protein [1] (Figure 1). In Colombia on March 29, was obtained the first SARS-
CoV2 genome sequence that circulates in Colombia, SARS-CoV2 arrives in the country on February 26, 2020.

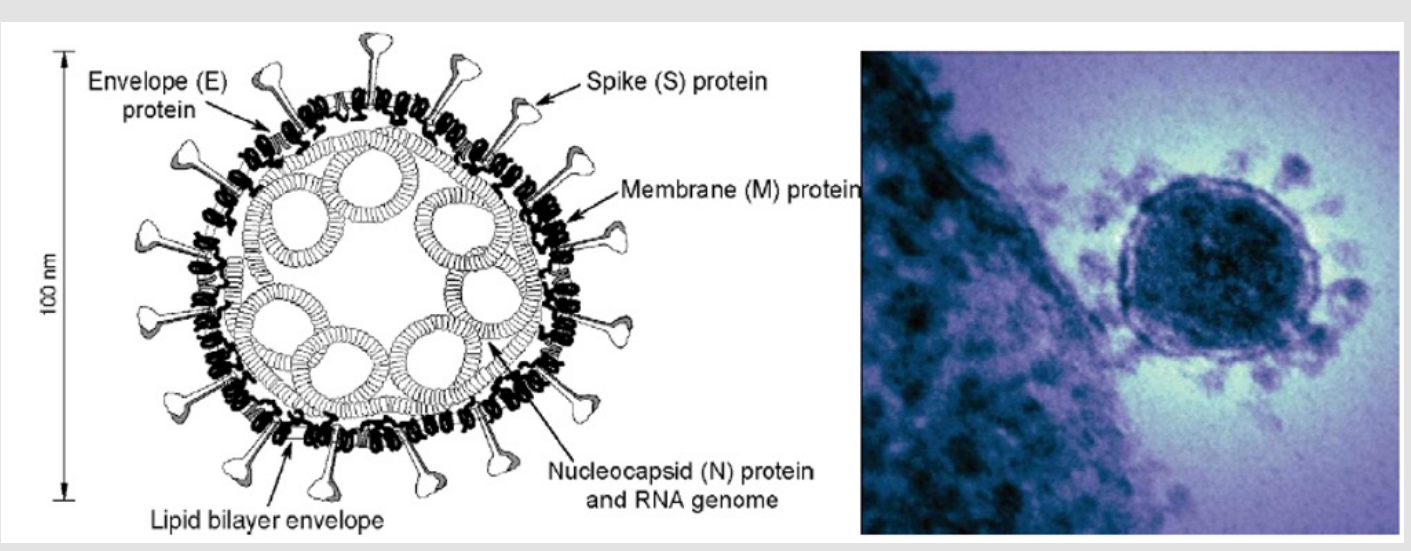

Figure 1: General morphology of coronaviruses, to the left microphotography of COVID-19 taken [1].

\section{Viral Replication, Cycle and Assembly}

The virus binds to the host cell by interacting with the Spike protein with the receptor of the cell to be infected, this receptor varies in the host, in humans two receptors have been described, the angiotensin-2 receptor (ACE2) and the human receptor of aminopeptidase-N N (hAPN). Once inside it deposits its RNA, moves to the ribosomes and from there begins the protein translation and finally its assembly as outlined in the Figure 2 [1]. The importance of knowing that one of the receptors is ACE-2 has largely explained the tropism that has had the virus by the respiratory system, cardiovascular and the possible explanation of their neurological commitment, They have even argued that the severity of the disease may be related to patients with cardiovascular disorders and the cytokine storm that it generates (IL-1B, IL-6, IL-12, IFN, IL-10, and MCP1) by a TH2 response [2,3] that are currently being worked on in clinical trials as blocking agents of this receptor in its therapy [4].

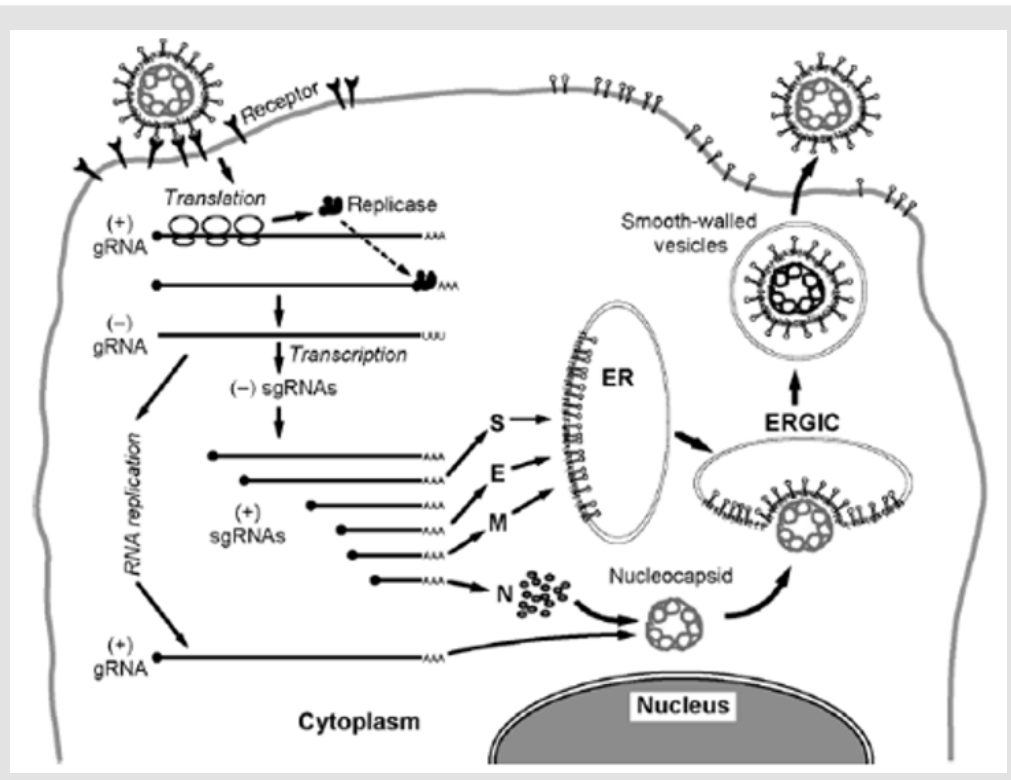

Figure 2: Image that outlines the viral cycle of coronavirus in the host cell, Taken from [1].

\section{Where Did it Come From?}

On 31 December 2019, an epidemiological alert was issued for an outbreak of pneumonia caused by a new strain of coronavirus that occurred in Wuhan in Hubei Province, China, with possible epicenter the Huanan wholesale seafood market, where wild animals were sold illegally (bats, badgers), which is considered to have originated from bats $[2,5,6]$. The first confirmed outbreak was
41 patients, isolating the new virus on 7 January calling it the new coronavirus of 2019 or $2019-\mathrm{nCoV}$, later renamed as severe acute respiratory syndrome coronavirus-2 (SARS-CoV-2) and the pathology generated was called Coronavirus Disease 2019, acronym COVID-19 $[7,8]$. Its spread was rapid to the point that in less than three months of the outbreak in China, the World Health Organization (WHO) declared COVID- 19 as a pandemic on 11 March 2020, being 
present in more than 110 countries with an estimated attack rate of $1 \%$, accounting for 118.322 infected patients and 4292 deaths [8].

\section{How is it Transmitted and What is Your Incubation Time?}

Transmission of the virus is by aerosols or drops, the secretions (saliva, feces, conjunctival) of the infected person or with exposure to an inert area or surface where the virus was [2,9], summarize as follows:

\section{Person-To-Person}

COVID-19 is transmitted by airway, fecal-oral and by contact of respiratory, conjunctival secretions, it has not been reported that sweat, seminal fluids or vaginal fluids transmit it.

\section{At the Respiratory Level}

The transmission is by inhalation of droplets containing the virus within a contact close to 6 feet or 2 meters away.

\section{Contact with Surfaces, Contaminated or Inert Objects [10].}

a) The droplets of 5 micrometers, maintain stability on the following surfaces or vehicles.

b) In the air in aerosol mode $3 \mathrm{~h}$.

c) Plastic, steel metal up to $72 \mathrm{~h}$.

d) Surfaces of copper (4h) and cardboard (8h).

It is therefore that COVID-19 has high propagation efficiency, reporting that by 1 infected patient it has already transmitted it to 2.2 patients [2], so preventive isolation means can decrease rapid growth in incidence. Its incubation period has been obtained in relation to SARS and MERS episodes with an average incubation time of 2-14 days after exposure to the vector $[6,11]$.

\section{How is it Diagnosed?}

The most relevant thing at the moment is the epidemiological nexus whether it is to travel in the last 14 days to areas where this virus or contact with infected people, then the presence or not of symptoms should be evaluated (see below), it is essential not to panic since the first error is to consult emergency department for mild symptoms, in case of alarm signs (see below) consult these services so as not to generate saturation in the services and promote transmission of the virus. Likewise, each institution must have areas specifically designated for patients with suspected COVID-19, transfer routes, fitting beds in the intensive care unit (ICU) for management, institutional route guides, boarding and creating teams of experts to lead decision-making in difficult situations and the whole group handles the same language and follow the same technical-scientific and ethical guideline.

\section{Symptoms of COVID-19 Infection}

The clinical spectrum of COVID-19 range from asymptomatic cases, mild rhinopharyngitis that account for approximately $80 \%$ of cases, to generating serious manifestations represented by $20 \%$ mainly pneumonia that will require hospitalization with some type of intervention, of which 5-8\% will require intensive care in which Acute respiratory distress syndrome (ARDS) will be their most serious complication in a $46-60 \%$ of cases. But not everything is bleak as the resolution in mild cases is very high up to $80 \%[12,13]$, so being at home if you have mild symptoms is the best way to help the health team mitigate the pandemic. Generally, symptoms may appear within 2 to 14 days of exposure to the virus and the three most common symptoms are:

1) $\mathrm{T}$ fever $38^{\circ} \mathrm{C}$ with axillary thermometer.

2) Dry cough.

3) Shortness of breath or difficulty breathing.

4) Others: myalgia, fatigue, chills, gastrointestinal (diarrhea and nausea).

If the person has ANY of the following symptoms or warning signs, he or she should have prompt medical attention or consult the emergency department.

1) Difficulty breathing.

2) Persistent chest pain or pressure.

3) Impairment in consciousness or inability to awaken.

4) Bluish lips or face.

5) Hemoptysis (cough with blood)

\section{What Symptoms Does the Medical Literature Re-} port?

From the population of Wuhan described by Huang et al [2], Wang et al [14] and Zhu et al [11], Guan et al [15] and Zhou et al [16] described that the symptoms and characteristics of the patients were:

\section{Demographics}

a) Similar rate in men and women ( $54 \%$ men).

b) Average age between $42-68$ years.

c) Transmission to health personnel was $29 \%$ and hospital patients were $12.3 \%$, implying that the $41 \%$ of cases are processed at the nosocomial level.

\section{The Most Common comorbidities Were}

a) Diabetes $10-20 \%$.

b) High blood pressure $15-30 \%$.

c) Cardiovascular disease $15 \%$

d) Cancer $5.6 \%$.

e) More than $50 \%$ of patients had no comorbidities $[2,11,14]$ (Table 1) 
Table 1: Signs and symptoms according to frequency.

\begin{tabular}{|c|c|}
\hline The Most Frequent & The Less Frequent \\
\hline Fever $98 \%$ & Palpitations $7-8 \%$ \\
\hline Fatigue $70 \%$ & Headache $6.5-8 \%$ \\
\hline Dry cough $60-76 \%$ & Hemoptisis $1-5 \%$ \\
\hline Myalgia $15-20 \%$ & Nausea and vomiting $2-5 \%$ \\
\hline Sputum production $26-28 \%$ & Diarrhea $2-5 \%$ \\
\hline Adenopathies $1 \%$ & \\
\hline
\end{tabular}

If we make a timeline like the one shown in Figure 3 regarding the development of symptoms since the onset of the disease it is described that admission to the hospital occurred on average at 7 days (1-8 days), dyspnea at day 8 (0-0 9 (0-14 days), need for mechanical ventilation 9-10 days (7-17 days) and admission to ICU on day 10-11 ( 8-17 days), gives us a picture to be able to evaluate the clinical evolution of these patients indicating that the course is stationary between 7-10 and after that time you can see the progression or improvement of the picture, it should be noted that the severity can be present from the beginning of the symptoms. Regarding hospitalization time in non-complicated cases were on average 10-12 days and in ICU 8-10 days [2,11,14,15,16] (Figure 3).

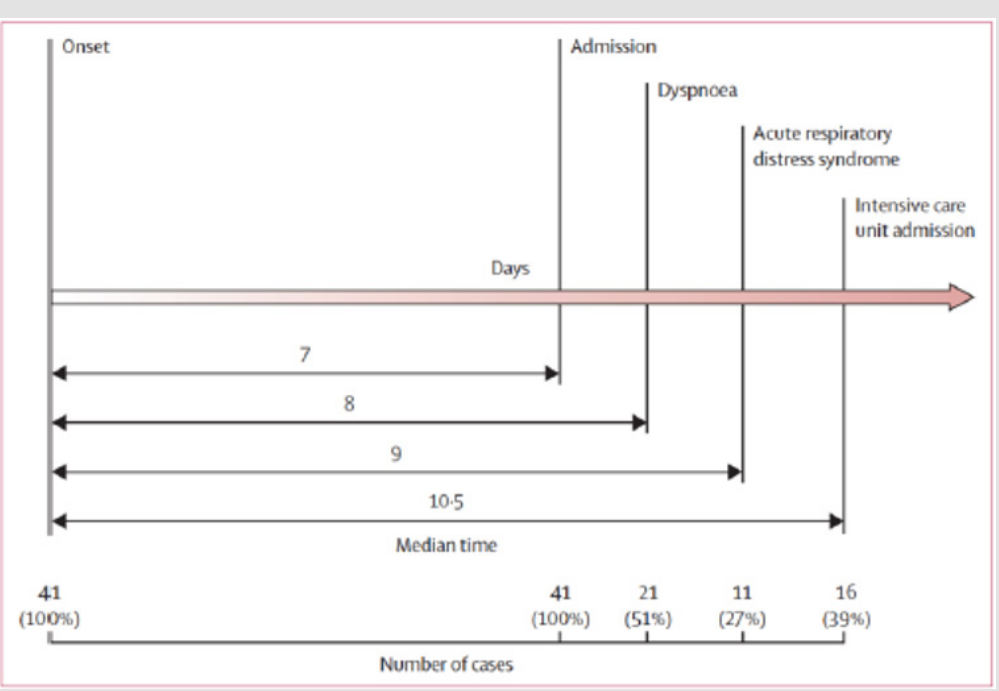

Figure 3: Timeline of symptom evolution of COVID-19 cases, taken from [2].

In the blood chemistry, The Most Common Abnormalities Were
a) Lymphopenia $(<1000 \times 10-9) 63-70 \%$.
b) Thrombocytopenia $<150,00036-40 \%$.
c) Prolonged activated partial thromboplastin time (PTT) test $58 \%$.

d) Elevated lactate dehydrogenase (LDH) $>240$ U/L: 40 $74 \%$.

e) C-reactive protein (CRP) elevated $>10 \mathrm{mg} / \mathrm{L} 40-60 \%$.

f) D-dimer $>500 \mathrm{mcg} / \mathrm{L} 3-2 \%$.

g) Transaminase elevation $>40 \mathrm{U} / \mathrm{L} 20-40 \%$.

h) Creatine kinase fraction MB (CK-MB) > $180 \mathrm{U} / \mathrm{L} 15 \%$.

It should be noted that, although not all of the following laboratories were statistically significant, having elevated D-dimer, lymphopenia, elevated LDH, CK-MB and elevated liver function was seen more in ICU patients, with an average admission SOFA of 5 points, indicating that these laboratories might indicate that patients would be more likely to have a more severe disease, emphasizing that lymphopenia could be considered a marker of this disease from baseline[2,11,14].

\section{Procalcitonin (PCT) and COVID-19}

The Huang and Wang studies (2.8) did not report PCT elevation, suggesting that if it rises in these patients it may be a sign of bacterial infection, which was seen at 25\% (PCT \%-0.5 ng/ml) in the Huang series and 75\% in Wang's respectively, indicating that the PCT may have the identification of bacterial infection in these cases.

\section{Radiologically}

Lung abnormalities can be identified in $86 \%$ of patients from the first day of admission to the hospital, where extensive pulmonary involvement is evident in chest x-ray in more than two quadrants, the most common patterns on pulmonary tomography finds were $[2,14,11,15,17]$.

a) Bilateral ground glass patchy opacities in peripheral or subpleural areas 95-98\%

b) Bilateral compromise of ground glass, without affecting central space $70-98 \%$

c) Multilobar consolidation in subpleural area $70-80 \%$ 
d) Other findings: Crazy paving pattern (thickening of interlobulillary septum with opacity with ground glass), subpleural reticular interstitial pattern, halo signs nodules, diffuse consolidation or bat sign. e) Findings to think in other etiologies instead of COVID-19: pleural effusion, lymphadenopathies, cavitation's, three in bud. (Figure 4).

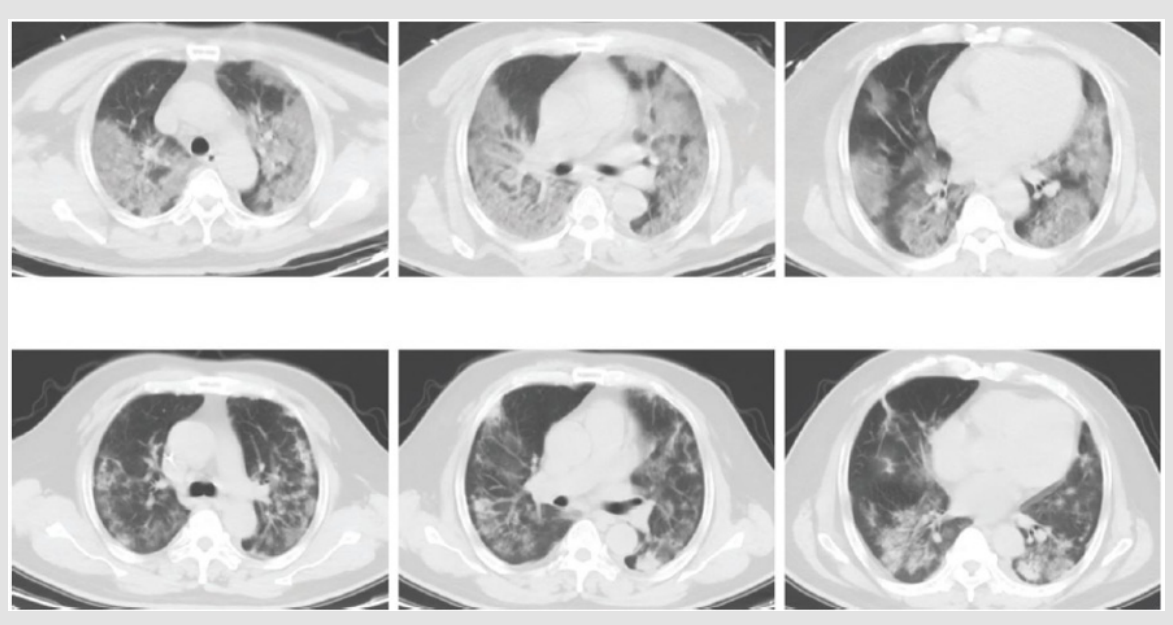

Figure 4 .

\section{POCUS and COVID-19}

Little have been reported from the POCUS (Point of care ultrasound) experience, in which pulmonary ultrasound with microconvex, convex or sectoral probe have been used to identify different patterns, in which pattern $B$, interstitial pattern, consolidation pattern has been described, it is important that, if this tool is available and no CT scanner is available, ultrasound is used.

\section{Place of Destination and Complications}

Among Huang and Wang's studies, the average number of patients who were hospitalized were mostly in general rooms
$65-70 \%$ and $25-30 \%$ in the ICU with age variation as outlined in Figure 4. Regarding its complications the most common were the development of ARDS, shock, heart injury and secondary infection, attributed by cytokine release syndrome (cytokine storm) varying in the groups of ICU vs No ICU (general room) (Figure 5) (Table 2).

Table 2: Complications in COVID-19 ICU vs No ICU patients.

\begin{tabular}{|c|c|}
\hline UCI Group & Non-ICU Group \\
\hline SDRA $61-85 \%$ & Arrhythmias 7\% \\
\hline Arrhythmias $44 \%$ & SDRA 5\% \\
\hline Shock $20-30 \%$ & Lesión cardiaca 2-4\% \\
\hline Acute heart injury 22-31\% & \\
\hline Sepsis, secondary infection 31\%. & \\
\hline
\end{tabular}

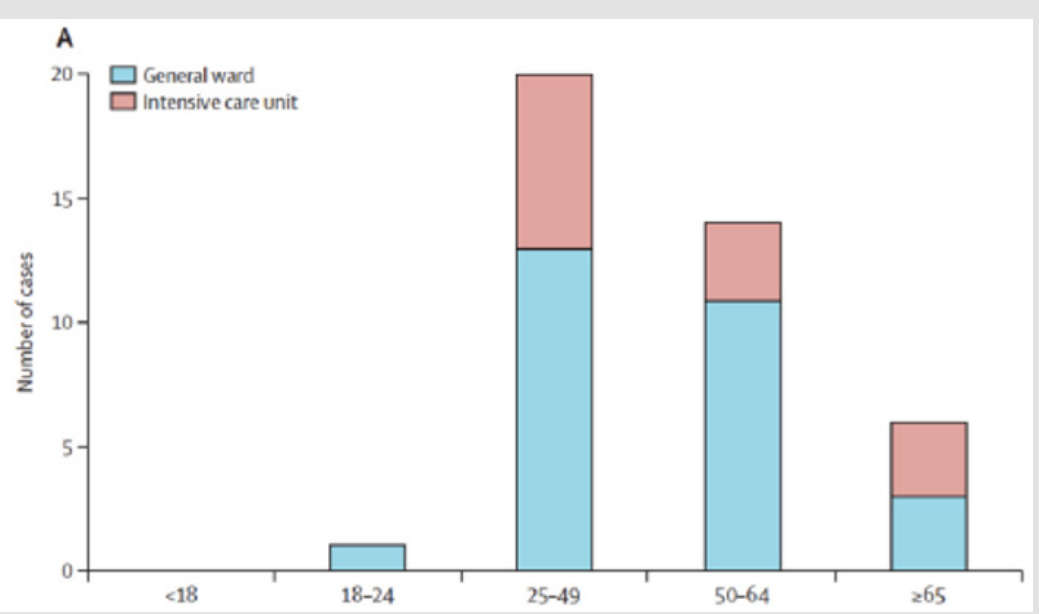

Figure 5: Location of patients hospitalized by COVID-19, distributed by age range. Taken [2].

\section{Cardiovascular involvement and COVID-19}

Coronaviruses have tropism and extensive cardiovascular disease in case of SARS and MERS with spectra of myocardial injury and acute or chronic heart failure, the various manifestations that have been reported in the Cases of Wuhan of COVID-19 reflect this same pattern $[2,11,14,15]$, a pattern manifested with development of arrhythmias, acute heart injury manifested with elevated troponin and $\mathrm{CPK}-\mathrm{Mb}$, even reporting that some patients consulted 
in the emergency room not for respiratory symptoms but by cardiovascular symptoms given by palpitations and chest pain [3] showing SARS-CoV2 may have clinical outcomes such as acute viral myocarditis , shock and acute heart failure $[3,18]$. It is important to consider the role of cardiology societies in managing patients with cardiovascular disease and COVID-19, in which Driggin and colleagues make an impeccable summary [6].

\section{Cardiovascular Spectrum and COVID-19 [6,16]}

a) Myocardial injury: elevation of cardiac biomarkers (troponin, CPK-MB).

b) Acute heart injury: elevation of biomarkers $>$ p99th + electrocardiographic and echocardiographic changes.

c) Arrhythmias: do not specify what type, but is considered in relation to metabolic, hypoxic and neurohumoral stress.

d) Acute heart failure: present in an average of $23 \%$ and more than half of patients who died in studies in China [16].

e) Risk of development of post-infection acute coronary syndrome.

f) Others: Fulminant viral myocarditis.

\section{Used Therapies Reported in Literature}

The experience that is had in the use of therapies is based on the report of the different series and therefore find levels of $\mathrm{AI}$ evidence, it will be very difficult, and we will guide ourselves with the recommendations of Class $C$ i.e. expert concept and evidence level II-III, which will be case reports, case series and some small cohorts From the previous what was reported has been:

a) Oxygen support.

i. Nasal cannula $66 \%$ or

ii. NIMV (Non-invasive mechanical ventilation) or HFCN (high-flow nasal cannula) 41\%.

iii. IMV (Invasive Mechanical Ventilation) $47 \%$ or

iv. $\quad$ ECMO 2-5\%

b) Antibiotics: $100 \%$.

i. Reported moxifloxacin, azithromycin, ceftriaxone.

c) Antivirals $89-93 \%$.

a. Oseltamivir $75 \mathrm{mg}(80-93 \%)$.

b. Lopinavir / ritonavir.

d) Systemic steroids 22-45\% (methylprednisolone).

e) Vasopressors 22\%.

f) Immunomodulators i. Immunoglobulin 13-24\%, tocilizumab, chloroquine, hydroxychloroquine.

g) Renal replacement therapy (RRT) 7\%.

\section{Prognosis}

At the moment unlike their relatives SARS and MERS who had a mortality of $11 \%$ and $35 \%$ respectively [2], COVID-19 mortality has been variable in WHO statistics, with $4.3 \%$ in Wuhan, China up to $8 \%$ in Italy with an average of $4.5 \%$ [12], of course these data must be constantly being monitored, but it is not a negligible mortality to be assessed in relation to population size, mentioning that the main patients at risk of mortality were those with cardiovascular comorbidity or have advanced age. An example of this is Colombia with an estimated population of approximately 50,000,000 habitants with a serious estimate of infection [17]. In Wuhan case studies, mortality in ICU averaged 38\% and non-ICU 15\% studies $[2,9,11]$.

\section{Case Definition (Colombian Guides- National Insti- tute of Health)}

Following guidelines similar to those of WHO, the National Institute of Health with the guidance 'GUIDELINES FOR THE DETECTION AND MANAGEMENT OF COVID-19 CASES BY HEALTH SERVICE PROVIDERS IN COLOMBIA VERSION 05, Ministry of Health and Social Protection Bogotá, March 19, 2020, classified in 5 cases the suspicion of patients with COVID-19 being these:

a) Case 1 Patient with unusual Severe Acute Respiratory Infection (USARI).

b) Case 2 Symptomatic non-serious patient who does NOT require hospitalization.

c) Case 3 Severe Acute Respiratory Infection (SARI) intensified surveillance for COVID-19 sampling.

d) Case 4 Probable death by COVID-19.

e) Case 5 Asymptomatic patient.

\section{It is also Essential to Define Close Contact}

a) Contact between two people in space less than $2 \mathrm{~m} / 6 \mathrm{ft}$ with a confirmed or probable case for 15 minutes

b) Having direct contact with secretions of a probable or confirmed case

\section{Cases are Defined as Follows}

\section{Case Criteria 1}

Probable Case: Patient with cough and fever, with severe acute respiratory infection box - SARI who develops an unusual or unexpected clinical course, especially a sudden deterioration despite proper treatment, requiring hospitalization (does not require travel history) (unexpected SARI). 


\section{Case 2 Criteria}

Probable Case: Person with at least 2 of the following symptoms: fever greater than or equal to $380 \mathrm{C}$, cough, shortness of breath, odynophagia, fatigue and also has one of the following (NO HOSPITALIZATION REQUIRED MANAGEMENT): a. Travel history or that you have lived in China or countries and areas with viral circulation confirmed of the new coronavirus (SARS-CoV-2) in the 14 days prior to the onset of symptoms, or b. An occupation as a health worker or other staff working in an environment that cares for IRAG patients with unknown etiology, or c. A history of close contact in the last 14 days with a probable case or confirmed of severe acute respiratory infection by the new coronavirus.

\section{Case 3 Criteria: Surveillance of severe acute respiratory infection (SARI) in hospitalized patient}

Person with acute respiratory infection (ARI) with a history of fever and cough no more than 10 days of evolution, requiring inhospital management, samples should be taken for COVID- 19 .

\section{Case Criterion 4: Probable death by COVID-19}

All deaths from severe acute respiratory infection with clinical picture of unknown etiology.

\section{Case Criterion 5: Asymptomatic case}

Narrow contact of confirmed case COVID-19 that has not developed symptoms in the first 7 days after the last unprotected exposure.

\section{Case classification}

Several revisions are there to classify severity, this review will take the classification of the Handbook of COVID-19 Prevention and Treatment, made by Chinese authors who handled COVID-19 infection [13], which offer simple criteria and the severity classification data have been taken based on them [15], however, there are other guidelines such as those of WHO [4].

\section{Mild Cases 80\%}

Clinical symptoms are mild, and no manifestations of pneumonia can be found in imaging.

\section{Moderate Cases}

Patients have symptoms such as fever and respiratory tract symptoms and may have manifestations of pneumonia in the images.

\section{Severe Cases 14\%}

a) Adults who meet any of the following criteria

1) Respiratory frequency $>=30$ breaths $/ \mathrm{min}$

2) Oxygen Saturations $<=93 \%$ at sea, in Bogota $<90 \% *$

3) Ratio $\mathrm{PaO}_{2} / \mathrm{FiO}_{2}<=300 \mathrm{~mm} \mathrm{Hg}$

4) Patients who more than $50 \%$ of lung injuries progressed within 24-48h.

\section{Critical Cases 5\%}

a) Meets any of the following criteria (Derive to Intensive Care Unit/COVID-19)

1) Appearance of Respiratory Failure Requiring Mechanical Ventilation

2) Presence of shock

3) Another organic failure that requires monitoring and treatment in the ICU

Critical cases are divided into early, middle and late stages according to the oxygenation rate and state of the respiratory system.

\section{Early Stage}

1) $\mathrm{PaO}_{2} / \mathrm{FiO}_{2}$ 150-100 mm Hg; Pulmonary distensibility $>$ at $30 \mathrm{ml} / \mathrm{cm}$ without organ insufficiency apart from the lungs.

2) It has a great possibility of recovery through active antivirals, cytokine anti-storm and supportive treatment.

\section{Middle Stage}

$\mathrm{PaO}_{2} / \mathrm{FiO}_{2}$ 100-60 mm Hg; Pulmonary distensibility 30-15 $\mathrm{ml} / \mathrm{cm}$ can be complicated by another type of mild to moderate dysfunction of other organs.

\section{Late Stage}

1) $\mathrm{PaO}_{2} / \mathrm{FiO}_{2}$ less than $60 \mathrm{~mm} \mathrm{Hg}$; Pulmonary distensibility less than $15 \mathrm{ml} / \mathrm{cm}$, diffuse consolidation of both lungs requiring the use of ECMO; Or failure of other vital organs.

2) The risk of mortality increases significantly.

\section{Addressing and Diagnostic Tools}

The first step is the triage system in which the physician trained to identify these patients, does so and provide them with a conventional eye cap, gloves and refer it to the designated isolation area for those patients where drop and contact protection must be present. If the patient is stable and without an alarm sign, he or she may be referred home and contacts his health insurance or National health institute (INS) for sampling, if the patient with alarm signs will be hospitalized, the samples for COVID- 19 established by nasal swab or pharyngeal, basic blood chemistry studies will be taken: blood count, clotting times, liver function, renal function, chest x-ray, electrolytes, LDH. In our medium for the diagnosis of COVID-19 is counted with the RT- PCR (real time- polymerase chain reaction) method, this sample is obtained from respiratory swab (nasal or pharyngeal) with an average process time of 3-4 days, is taken in the clinic where the case is and is derived to the INS laboratory. It is important to mention that this method is not $100 \%$ sensitive, it has a sensitivity of $61-71 \%$ implying a margin of false negatives of $29-30 \%$, recently published as the use of tomography could supplement and rectify false negatives by the RTPCR method, indicating that $93 \%$ of patients may have a suspicious 
chest tomography before the result of the chest-PCR [19,20]. In case the patient arrives with indication of orotracheal intubation (see below) it should be referred to the intensive care unit or isolation unit designated in order to perform the procedure and mitigate the biological risk.

\section{What Protective Measures to Take?}

In general, the most important protective measures to be taken are:

1) Be quarantined during the pandemic: leave alone if necessary, if infected DO NOT leave, designate someone for food.

2) Proper hand washing as it reduces transmission of the virus by $50 \%$.

3) If you are infected with COVID-19, remain in strict quarantine.

\section{Protective Measures at Home}

a) The insulation of the carrier case applies to grooming, clothing, places that are exposed.

b) Hand washing with soap water for at least 60 seconds or with alcohol-concentration gel $60 \%$.

c) Hand washing should be performed.

1) After blowing your nose, coughing or sneezing o After using the bathroom

2) Before eating or preparing food.

3) After contact with animals or pets.

4) Before and after providing routine care to another person who needs assistance (for example, a child)

d) Use of chlorinated solutions to clean surfaces

1) 1 gallon of water (3.8 liters of water) +5 tablespoons of chlorine or $1 / 3$ cup is mixed so that a mixture with the eradication capacity of the virus remains.

2) This mixture can be packaged in a disperser or atomizer to be applied to surfaces.

e) Avoid contact of the hands with mucous membranes (eyes, nose, mouth).

f) Home care INS guidelines or

https://www.minsalud.gov.co/Ministerio/Institucional/ Procesos\%20y\%20procedimientos/GIPG13.pdf

g) Home isolation INS guidelines or

https://www.minsalud.gov.co/Ministerio/Institucional/ Procesos\%20y\%20procedimientos/GIPS06.pdf.

\section{Protection Measures Away from Home}

a) Ideally not to leave unless strictly necessary, local or national lines should be followed when the quarantine is established.

b) Bottom line: Better to stay home and not go out.

\section{Protective Measures in Hospitals}

a) Recommendation of personal protective elements (PPE) according to the level of exposure or https://www.minsalud. gov.co/sites/rid/Lists/BibliotecaDigital/RIDE/VS/tabla-eppconsenso.pdf

b) Handwashing with soap and water in 5 moments or using alcohol of $60 \%$ concentration.

c) Patients with probable, suspected or confirmed cases should be isolated using aerosol and contact protection measures

1) The sick must wear a mask.

2) Have a default route for the patient to minimize patient exposure with the medium.

\section{Personal Health Biosecurity Equipment}

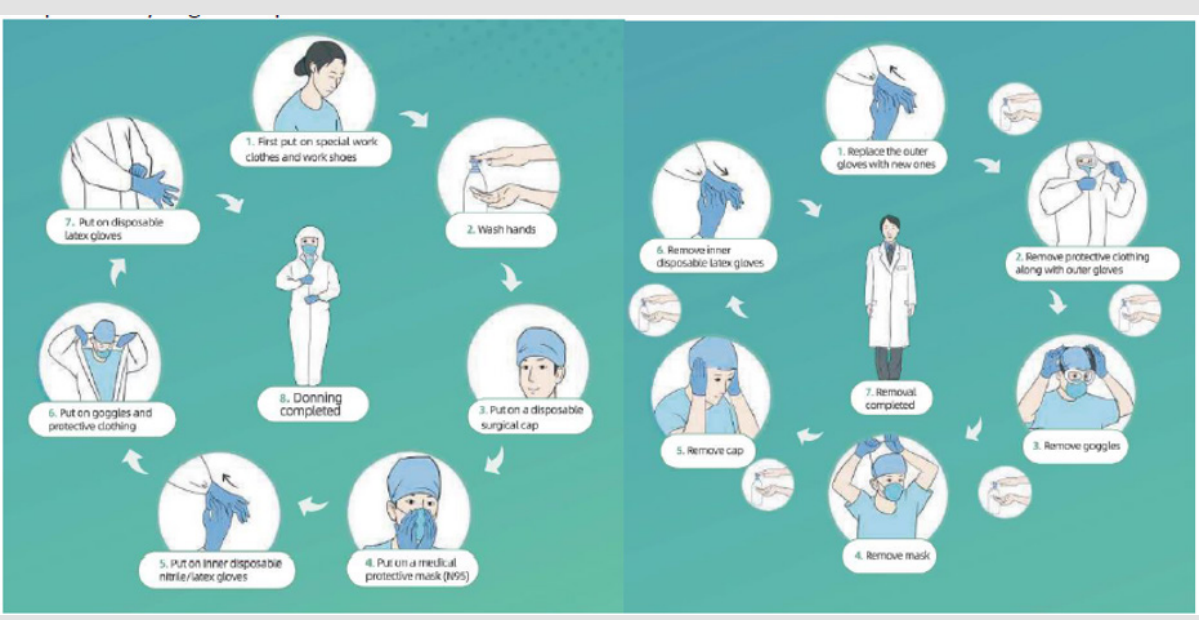

Figure 6. 
a) High-efficiency masks FFP2 (In the case of procedures with risk of aerosolization)

b) Eye protection with goggles

c) Long-sleeved clean bat.

d) Use double glove if in contact with coVID-19 patients.

The following illustrations from reference 12 illustrate how-to put-on PPE and how to remove them, remember to be patient, and follow the steps (Figure 6).

\section{Handling and Disposition of Corpses}

a) The Ministry of Health already has guidelines for how to gag, to manage the patient's corpse with COVID-19 on the following link:

b) https://www.minsalud.gov.co/sites/rid/Lists / BibliotecaDigital/RIDE/VS/ED/VSP/orientaciones-handlingcadaveres-covid-19.pdf

c) The most relevant here the person who performs it must ALWAYS maintain the high-efficiency head covering and the hospital where they work must already have the route of these patients established.

\section{Special Population}

\section{Mothers and COVID-19 (19)}

a) Few studies have described this relationship at the time only a series of 9 maternal cases have been reported in the third trimesters, in which the clinical course was similar to that described in the general population in relation to the clinic concluding from this report:

1) No fetus-placental transmission of COVID-19 was demonstrated, not found in breast milk.

2) It is not established if there is vertical transmission since all deliveries were by caesarean section.

3) None of the maternal ones died or severe SDRA development.

4) There were no postpartum complications of the newborn or alterations in APGAR.

b) WHO recommends the support measure as the leading therapy in this group of patients [17]?

\section{Treatment}

There is currently no specific treatment or proven vaccine for any coronaviruses or that are endorsed by regulatory bodies such as FDA, EMA or INVIMA in our country, however it is emphasized that if we expect to find treatments with AI level of evidence (randomized studies, double-blind, meta-analysis) will be very difficult to adjudicate the effectiveness of a treatment in these times, that is why we must go to gray literature or what at a level of recommendation will be $\mathrm{C}$ (small observational studies, expert opinion), with Class II-III evidence (series of cases, cases and controls or small cohorts), the most important thing is to follow the guidelines of ethics and the principle of non-maleficence. Available pharmacological treatments have been demonstrated in in vitro studies, few in vivo, through small series of cases and controls, describing as objectives: reducing the amount of circulating RNA (RNAemia), impacting clinical outcomes and impact on mortality, that at the moment none of the pharmacological therapies to be mentioned below to complicate all of these objectives, the Atlanta CDC on its page (https://www.cdc.gov/coronavirus/2019-ncov/ hcp/therapeutic-options.html) mentions some pharmacological therapies and may serve as a reference with WHO to consider the use of certain therapies [4,21]. The main current management is supportive and biosecurity therapy (contact and droplets, oxygen therapy, symptomatic management), below will describe what has been used and reported with more clinical involvement, but many therapies have been described experimentally [6] The approach may be made if the patient has mild-moderate symptoms or has signs of severe acute respiratory infection (IRAG), the latter outlines WHO guidelines in its COVID-19 severe respiratory infection management guide [21].

\section{Isolation and Biosecurity Measures}

a) It is the first tool to be implemented with isolation of the patient with droplets and contact, in a unit where only those cases are, to mitigate hospital transmission.

b) Implement special routes for these patients.

c) Safe sample handling.

\section{Oxygen Therapy}

a) Oxygen substitution is the first most commonly used pharmacological measure in descriptive studies, with indication of nasal cannula when oxygen saturation $\left(\mathrm{SatO}_{2}\right)<=90 \%$ or a $\mathrm{PaO}_{2} / \mathrm{FiO}_{2}<=300$ with a $\mathrm{SatO}_{2}$ goal, $90 \%$ in non-pregnant women and $>=92-95 \%$ [21].

b) if $\mathrm{PaO}_{2} / \mathrm{FiO}_{2}$ between 200-300 mmHg the use of HFNC with flow of 50-60 L/min can be considered.

\section{IOT indications [13]}

a) $\mathrm{PaO}_{2} / \mathrm{FiO}_{2}<150 \mathrm{mmHg}$ in patients under 60 years and over 60years $\mathrm{PaO} 2 / \mathrm{FiO} 2<200 \mathrm{mmHg}$

b) Respiratory frequency < of $30 /$ minute

c) Glasgow comma scale $=<8$

d) $\mathrm{CO}_{2}$ greater than $50 \mathrm{~mm} \mathrm{Hg}$

e) Respiratory clinical course impairment whit signs of multiple organ failure following therapy with another oxygenation medium. 


\section{Invasive Mechanical Ventilation}

In this scenario the patient must managed by an expert in this subject, since it is possibly found in a case of ARDS (acute respiratory distress syndrome) but the following parameters can initially be adjusted by no non-expert personal

a) Ventilation Mode: Volume-Controlled Assist-Control Mode (A/C).

b) Current or tidal volume (VC/VT) $6-8 \mathrm{cc} / \mathrm{kg}$ by ideal weight.

a. Adjust according to peak pressure and pulmonary distensibility

b. Predicted Weight:

c. Male: $50+0.91$ (height-154).

d. Woman: 45.5+9.91(height-152.4).

c) $\mathrm{SotO}_{2}$ Goals: $86-90 \%$ or

d) Initial PEEP 8-12 $\mathrm{cm} \mathrm{H}_{2} \mathrm{O}$, pitiable according to the method that has the most knowledge (distensibility, driving pressure, ultrasound, etc.).

a. Objective of the PEEP: maintains alveolar recruitment and facilitates the exchange Pulmonary

e) Respiratory rate $12-25$, higher generates air entrapment

f) Ratio I: E 1:2-3

g) FiO2: initially high, but carry $60-50 \%$ to mitigate adverse oxygen events

h) Peak pressure and plateau press $<30 \mathrm{~cm} \mathrm{H}_{2} \mathrm{O}$

i) Driving pressure $<15 \mathrm{~cm} \mathrm{H}_{2} \mathrm{O}$

\section{Other Therapies}

1) Pronation and ECMO: topic to be determined at each institution

2) Pronation: consider early pronation $\left(\mathrm{PaO}_{2} / \mathrm{FiO}_{2}\right.$ ratio 140 $150 \mathrm{mmHg}$ ), $18 \mathrm{~h}$ of prone position rest $6 \mathrm{~h}$.

\section{Antibiotics}

a) It is vitally important that having COVID-19 is not an indication of antibiotic therapy especially in mild to moderate cases. The indication to be evaluated in severe cases where bacterial overinfection is suspected in which the therapy to be chosen will be in relation to the local guides of each hospital or having as a line the national guidelines of community-acquired pneumonia [22], remembering that before considering the initiation of antimicrobial therapy, take blood cultures, Gram samples and cultures of secretion/sputum, procalcitonin, so to be able to perform an effective management of antibiotics and remove it appropriately since ideally they will be removed in less than 48-72 if there is no documentation of bacterial infection.

b) In case of macrolide association associate management with azithromycin $500 \mathrm{mg}$ day 1 , then $250 \mathrm{mg}$ day for 5 days, with strict QT surveillance recommendation.

c) In the case of the patient with unexpected SARI following the national lines should be initiated with oseltamivir $75 \mathrm{mg}$ every $12 \mathrm{~h}$ for 5 days, in relation to influenza virus or H1N1, whit the subsequent rapid molecular testing such as PCR or the one available at your institution to identify such coinfection and thereby determine the continuity of the antiviral.

\section{Antivirals and SARS Cov-2}

a) As previously mentioned, no antiviral is approved by FDA, EMA or INVIMA for the management of SARS-Cov-2, but due to the rapid progression of the disease SARS and MERS data have been taken to indicate antiviral therapy in SARS-Cov-2.

b) The main objective of antiviral therapy is to reduce viral load and thereby mitigate severe clinical outcomes of the virus, currently data from remdesivir, lopinavir/ritonavir, Darunavir/cobicistat.

c) Remdesivir: is a nucleoside analogue with in vitro and in vivo activity against COVID-19, which is incorporated into viral RNA generating an early termination of coVID-19.

1) Posology: $200 \mathrm{mg}$ IV D1 then $100 \mathrm{mg}$ day IV for 5-10 days.

2) Currently several experts consider it if available as a first line, their evidence in combination with other therapies such as antimalarials have shown an impact on outcomes described above [23].

3) Interactions: Relevant since it has CYP3A4 system.

4) Adverse events: hypotension during infusion, not in patients with CHILD C or TFG $<30 \mathrm{~mL} / \mathrm{min} / 1.73 \mathrm{~m}^{2}$.

5) Ongoing studies: NCT04252664, NCT04257656.

\section{Ritonavir/lopinavir Tab $200 \mathrm{mg} / 50 \mathrm{mg}$ or Sol Oral $(80 \mathrm{mg} / 20 \mathrm{mg}) / \mathrm{ml}$}

a) Posology: $400 \mathrm{mg} / 100 \mathrm{mg}$ twice daily (two capsules or 5 $\mathrm{mL}$ VO or per SNG for up to 14 days

b) Recently the NEJM public a study of control cases where the mortality rate at 28 days was numerically lower with intervention vs standard management $(19.2 \%$ vs 25$)$ but had no statistical significance [24].

c) Interactions: inhibits the CYP3A4 system

d) Adverse events: QT prolongation being watching you, Gastrointestinal (nausea and diarrhea), hypertriglyceridemia 


\section{Immunomodulatory Therapy}

\section{Hydroxychloroquine and Chloroquine}

a) Alters the $\mathrm{pH}$ of the endosome it requires for the fusion of the virus with the cells, and they also describe mitigating cytokine release syndrome.

b) Hydroxychloroquine $[25,26]$.

1) Posology: Loading dosage $400 \mathrm{mg}$ every $12 \mathrm{~h}$ day 1 , then $200 \mathrm{mg}$ BID for 5 days

a) Ongoing studies: ChiCTR2000031174, NCT04261517

c) Chloroquine Phosphate Tab $250 \mathrm{mg}(150 \mathrm{mg}$ chloroquine base)

1) Posology: $500 \mathrm{mg}$ every $12 \mathrm{~h}$ for $5-10$ days

2) In the FAZHU hospital experience the scheme used was $500 \mathrm{mg}$ BID for 2 days, then $500 \mathrm{mg}$ day for 5 days [13].
3) Ongoing
studies:
ChiCTR2000031204,
ChiCTR2000030718

d) Interactions: Relevant as both inhibits inhibits CYP2D6

e) Adverse events: watch QT, documented macular toxicity and atrial blockage are long but surveillance in both molecules of QT.

\section{Glucocorticoids}

a) Its use is controversial and not proven to be clear benefit, the main argument is the delay of the purification of RNAI, in addition the presence of H1N1 infection should be ruled out already if increased mortality is administered [27].

b) Indications follow in relation to refractory shock, presence of ASTHMA or COPD

c) Posology: $1-2 \mathrm{mg} / \mathrm{kg} /$ day per 5 days

d) Steroid studies and COVID-19

underway: ChiCTR2000029386

\section{Conclusion}

Knowledge of respiratory disease caused by SARS-CoV-2 is constantly evolving, so it is necessary to stay up to date on the results of developing clinical trials and real-world experience to match therapy for the greatest benefit of patients. In the same way it is necessary to consider the extrapulmonary manifestations (cardiovascular, digestive, neurological, among others) to recognize complications early. Regarding treatment, it should be guided by institutional schemes and based on the body of evidence available pending further global clinical data. Finally, it is important to emphasize the vital importance of population prevention measures in order to reduce transmission chains, prevent the collapse of health systems and thus alleviate the morbidity resulting from this infection.

\section{Competing Interests}

The author declare that they have no competing interests.

\section{Acknowledgement}

To the effort made by the COVID-19 unit in the Hospital Universitario Mayor, Mederi, Bogota Colombia.

\section{References}

1. Masters PS (2006) The Molecular Biology of Coronaviruses. Vol. 65, Advances in Virus Research. Academic Press pp. 193-292.

2. Huang, Yeming Wang, Xingwang Li, Lili Ren, Jianping Zhao, et al. (2020) Clinical features of patients infected with 2019 novel coronavirus in Wuhan, China. Lancet 395: 497-506.

3. Zheng Y, Yi-Tong Ma, Jin-Ying Zhang, Xiang Xie (2020) COVID-19 and the cardiovascular system. Nat Rev Cardiol.

4. Ensayos clinicos en curso COVID-19. Estudios Chinos.

5. Emergencia en china por nuevo coronavirus.

6. Elissa Driggin, Mahesh V Madhavan, Behnood Bikdeli, Taylor Chuich, Justin Laracy, et al. (2020)Cardiovascular Considerations for Patients, Health Care Workers, and Health Systems During the Coronavirus Disease 2019 (COVID-19) Pandemic, Journal of the American College. of Cardiology.

7. (2020) Coronaviridae Study Group of the International Committee on Taxonomy of Viruses. The species Severe acute respiratory syndromerelated coronavirus: classifying 2019-nCoV and naming it SARS-CoV-2. Nat Microbiol.

8. (2020) Declaración de OMS: COVID-19 es una pandemia WHO Situation Report.

9. Reporte No 7 de situación de COVID 19 por OMS.

10. Van Doremalen N, Bushmaker T, Morris DH, Holbrook MG, Gamble A, et al. (2020) Aerosol and Surface Stability of SARS-CoV-2 as Compared with SARS-CoV-1. N Engl J Med.

11. Zhu, Dingyu Zhang, Wenling Wang, Xingwang Li, Bo Yang, et al. (2020) A Novel Coronavirus from Patients with Pneumonia in China, 2019. N Engl J Med 382: 727-733.

12. (2019) Casos de OMS de COVID-19, consultado 20 de marzo.

13. Handbook of COVI0-19 Prevention and Treatment.

14. Wang, Bo Hu, Chang Hu, Fangfang Zhu, Xing Liu, et al. (2020) Clinical Characteristics of 138 Hospitalized Patients With 2019 Novel Coronavirus-Infected Pneumonia in Wuhan, China. JAMA 323(11): 1061-1069.

15. Guan, Zheng-yi Ni, Yu Hu, Wen-hua Liang, Chun-quan Ou, et al. (2020) Clinical Characteristics of Coronavirus Disease 2019 in China. N Engl ] Med.

16. Fei Zhou, Ting Yu, Ronghui Du, Guohui Fan, Ying Liu, et al. (2020) Clinical course and risk factors for mortality of adult inpatients with COVID-19 in Wuhan, China: a retrospective cohort study . Lancet 395(10229):10541062.

17. Clinical characteristics and intrauterine vertical transmission potential of COVID-19 infection in nine pregnant women: a retrospective review of medical records.

18. Tian-Yuan Xiong, Simon Redwood, Bernard Prendergast, Mao Chen (2020) Coronaviruses and the cardiovascular system: acute and longtermimplications. Eur Heart J.

19. DANE poblacion colombiana.

20. Ai T, Yang Z, Hou H, Zhan C, Chen C, et al. (2020) Correlation of Chest CT 
and RT-PCR Testing in Coronavirus Disease 2019 (COVID-19) in China: A Report of 1014 Cases. Radiology: 200642.

21. (2020) World Health Organization. (2020). Clinical management of severe acute respiratory infection when novel coronavirus (2019-nCoV) infection is suspected: interim guidance, 28 January 2020. World Health Organization.

22. (2013) Recomendaciones para el diagnóstico, tratamiento y prevención de la neumonía adquirida en la comunidad en adultos inmunocompetentes. Infectio 17(Supl 1): 1-38.

23. Manli Wang, Ruiyuan Cao, Leike Zhang, Xinglou Yang, Jia Liu, et al. (2020) Remdesivir and chloroquine effectively inhibit the recently emerged novel coronavirus (2019-nCoV) in vitro.Cell Res 30(3): 269-271.

24. A Trial of Lopinavir-Ritonavir in Adults.

25. Philippe Gautret, Jean-Christophe Lagier, Philippe Parola, Van Thuan Hoang, Line Meddeb, et al. (2020) Hydroxychloroquine and azithromycin as a treatment of COVID-19: results of an open-label non-randomized clinical trial. International Journal of Antimicrobial Agents.

26. Yao X, Ye F, Zhang M, Cui C, Huang B, Niu P, et al. (2020) In Vitro Antiviral Activity and Projection of Optimized Dosing Design of Hydroxychloroquine for the Treatment of Severe Acute Respiratory Syndrome Coronavirus 2 (SARS-CoV-2). Clin Infect Dis.

ISSN: 2574-1241

DOI: $10.26717 /$ BJSTR.2020.27.004484

Juan Sebastian Espinosa-Serna. Biomed J Sci \& Tech Res

cC) This work is licensed under Creative

Submission Link: https://biomedres.us/submit-manuscript.php
27. Han K, Ma H, An X, Jing Chen, Zhiyong Lian, et al. (2011) Early use of glucocorticoids was a risk factor for critical disease and death from pH1N1 infection. Clin Infect Dis 53: 326-333.

Videos informativos e información adicional

Jornada SEIMC de COVID-19.

COVID-19 y embarazo.

Imágenes en COVID-19.

https://www.youtube.com/watch?v=9gJV2NcVcps\&feature=push-usub\&attr_tag=cZfT0Q-QhXJ0207T\%3A6

https://www.youtube.com/watch?v=bKJVYkAAogI\&feature=push-usub\&attr_tag=SVczckSAAr0PvrmL\%3A6

Metodos de higiene CDC.

https://www.youtube.com/watch?v=LAwfRkBtFAo\&feature=push-usub\&attr_tag=fnNFj4hV3G4SDzLE\%3A6

Primer reporte de COVID-19.

Curso OMS COVID-19.

POCUS y COVID-19

$\begin{array}{ll}\text { BIOMEDICAL } & \text { Assets of Publishing with us } \\ \text { RESEARCHES } & \text { - Global archiving of articles } \\ \text { - Immediate, unrestricted online access } & \text { - Rigorous Peer Review Process } \\ & \text { - Authors Retain Copyrights } \\ \end{array}$

